\title{
Appendicular mucinous adenocarcinoma associated with pseudomyxoma peritonei, a rare and difficult imaging diagnosis
}

\author{
Romeo Ioan Chira ${ }^{1}$, Codrut Cosmin Nistor-Ciurba ${ }^{2}$, Andreea Mociran ${ }^{1}$, Petru Adrian Mircea ${ }^{1}$
}

${ }^{1} 1^{\text {st }}$ Medical Clinic, Gastroenterology Department, Emergency Clinical County Hospital, ${ }^{2}$ Oncology Institute "Prof. Dr. Ion Chiricuţă", "Iuliu Haţieganu” University of Medicine and Pharmacy, Cluj-Napoca, Romania

\begin{abstract}
Pseudomyxoma peritonei (PMP) is a rare disease, caused by primary mucinous tumors that arise most frequently from appendix, ovary, or pancreas. Usually diagnosis is made by computed tomography, but ultrasonography can be a very useful imagistic method, if this diagnosis is taken into account by the observer. We present a case of a PMP caused by an appendiceal mucinous carcinoma, in a 34-year-old male patient, with family history of malignancies, diagnosed in our department. He was thereafter surgically treated - appendiceal resection, peritoneal lavage - followed by chemotherapy. We underline the importance of ultrasonography, even though at first encounter, the diagnosis of PMP being generally difficult.
\end{abstract}

Keywords: appendicular mucinous adenocarcinoma, pseudomyxoma peritonei, ultrasonography.

\section{Introduction}

Pseudomyxoma peritonei (PMP) is a rare disease, caused by primary mucinous tumors that arise from different sites, most frequently appendix, ovary, and pancreas. In rare cases, other sites are primarily involved (gallbladder, stomach, colorectum, fallopian tube, urachus, lung, and breast). The overproduction of mucin is followed by peritoneal dissemination and multifocal cellular implants on the peritoneal serosa. Accumulation of intraperitoneal mucin increases intra-abdominal pressure, with symptoms of bowel obstruction, dyspnoea, and malnutrition. The annually incidence is evaluated to 1-2 cases per million [1].

We present here a case of PMP with characteristic peritoneal lesions caused by an mucinous appendicular neoplasm in a young patient.

Received 12.09.2015 Accepted 15.10.2015

Med Ultrason

2016, Vol. 18, No 2, 257-259

Corresponding author: Romeo Chira, MD, PhD

1 st Medical Clinic

3-5 Clinicilor street

400006, Cluj-Napoca, Romania

Phone: +40264592771/1172

E-mail: romeochira@yahoo.com

\section{Case report}

A 34-year-old male man with a three months history of abdominal increased girth, abdominal pain in multiple quadrants, asthenic syndrome, and irritability was referred to our department. From his personal history we recall childhood-onset epilepsy with posttraumatic trigeminal neuralgy and right glenuhumeral subluxation. The patient was admitted earlier in a county hospital with the same complaints and an abdominal computed tomography (CT)-scan suspected PMP (fig 1), possibly with an appendix mucocele as primary

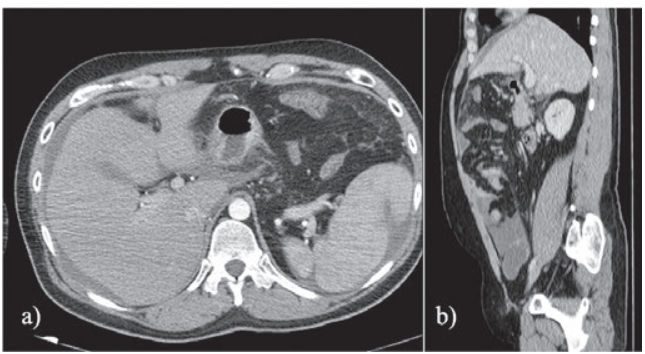

Fig 1. CT findings: a) upper abdominal transverse section (with contrast) showing peritoneal effusion surrounding liver and spleen, with indentations on the splenic diaphragmatic face; b) abdominal sagittal section with a cystic lesion in the right iliac fossa. 


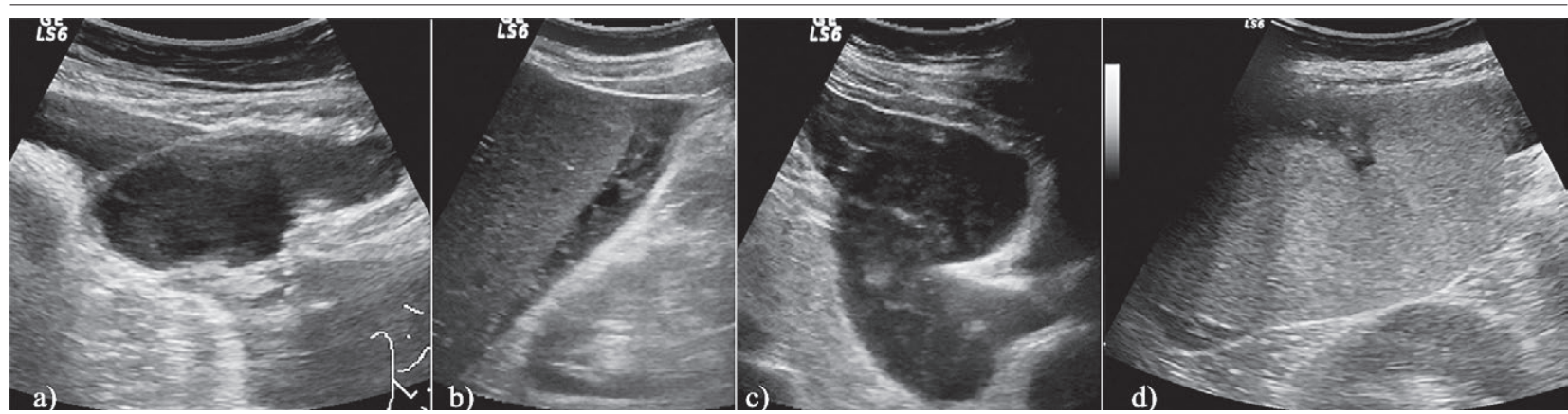

Fig 2. Ultrasonography findings: a) In the right iliac fossa a cystic appendiceal image thin but irregular wall, accompanied by ascites; b) Right hypocondrium section presenting interhepatorenal effusion with echogenic floating images; c) Ascites with echogenic nodules in the left iliac fossa; d) Irregular splenic surface, due to echogenic nodules inside the peritoneal effusion.

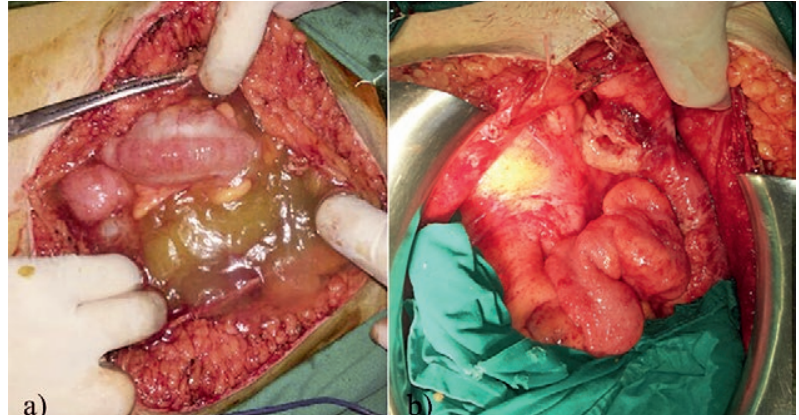

Fig 3. Intraoperatory image of the cystic tumor of the appendix and yellowish mucinous peritoneal effusion (a) and of the pseudomyxoma peritonei with nodular lesion (b).

tumor and was referred to our tertiary care center for complete evaluation.

Physical examination revealed pallor, globulous, distended abdomen, diastasis recti, sensitivity at abdominal palpation in iliac fossa, periumbilical, and hypogastric region. The laboratory workup showed mild anemia, inflammatory syndrome, and mixed dyslipidemia.

Abdominal ultrasound revealed a cystic tumor with peritoneal collection in the right iliac region. The effusion was inhomogeneous, with hyperechoic nodules that compressed the spleen surface (fig 2). Ultrasonographic findings were similar with the abdominal CT-scan findings. Therefore, a cystic tumor of the appendix with PMP was suspected.

For the investigation of the primary tumor upper and lower endoscopic examinations were carried out. Examinations revealed chronic erosive gastroduodenitis and small colonic polyps that were endoscopically removed.

Faced with a high suspicion of PMP most likely originated from an appendicular adenocarcinoma, we considered necessary the diagnostic laparotomy and surgical treatment. At the opening of the abdomen an important mucocele of the appendix was found (fig 3) occupying the right side of Douglas pouch, right iliac fossa and inferior aspect of the right flank making difficult the removal of the appendix.
Together with the wash-out of the ascites the removal of the classic peritoneal lesions of PMP was performed.

Histopathology report revealed a well differentiated (low grade) appendicular adenocarcinoma and it confirmed the diagnosis of PMP.

The patient made an uneventful recovery and was discharged in a stable condition.

After three weeks recovery the patient underwent chemotherapy.

\section{Discussions}

The 2010 WHO classification recognizes three main categories of appendicular mucinous neoplasms: mucinous adenoma, low-grade appendiceal mucinous neoplasms (LAMN) and appendiceal adenocarcinoma [2]. Also according to the same classification, PMP is classified into low-grade PMP, which is associated with LAMN, and high-grade PMP, associated with mucinous adenocarcinoma [2].

Disseminated peritoneal adenomucinosis is a pathological entity which has different histologic and gross features compared with peritoneal mucinous carcinomatosis. The prognosis of those diseases is also different. Disseminated malignant peritoneal disease has 5-year survival rates of $44 \%$, whereas low-grade peritoneal disease has been associated with $86 \%$ survival [3].

Ultrasonographic appearance of the peritoneal adenomucinosis is usually represented by a moderate amount of ascites, containing septation and echoes. Other signs can be present: scalloping of visceral surface, invasive parenchymal nodules, and peritoneal masses, in all types of mucinous neoplasm, but they are more frequent in carcinomatosis [4]. One of the most specific signs is the presence of hypoechoic areas in the thickened peritoneum [5]. The presence of an enlarged hypoechoic omentum (omental cake) or enlarged celiac lymph nodes, suggest peritoneal mucinous carcinomatosis [4]. In our 
case, we encountered a medium amount of ascites with echogenic pseudonodules and peritoneal nodules compressing the spleen. Also the primary cystic tumor of the appendix was very well observed by US. US can have an important role in PMP diagnosis if the suggestive signs of peritoneal mucinous effusion and lesions are visible, along with the primary tumor - usually with cystic appearance.

Ultrasound guided biopsy and drainage has been performed in many cases, but aspiration is inefficient in most situations, due to the particular consistency of the mucinous collection. In our case, with a high suspicion of a PMP of appendicular origin and considering the literature data [4], we chose not to perform the peritoneal puncture/biopsy.

CT and Magnetic Resonance Imaging (MRI) are helpful for the diagnosis of appendiceal mucinous neoplasms and PMP. Multidetector CT is considered the best imagistic method for the diagnosis of the pathology of ileocecal region [6]. If the appendix has a diameter of more than $15 \mathrm{~mm}$, the presence of a soft tissue mass, the thickening or irregularity of the wall, must raise the suspicion of a mucinous neoplasm $[5,7,8]$. On T2-weighted MRI images mucinous neoplasms can be seen as hyperintense tubular distention of the appendix [9].

The treatment combines aggressive cytoreductive surgery (CRS), aiming complete tumor removal, along with loco-regional heated chemotherapy to treat microscopic residual disease, namely hyperthermic intra-peritoneal chemotherapy (HIPEC) [10-12].

For patients treated by CRS combined with HPEC, the 5 -year survival rate varies from $74 \%$ to $100 \%$ for disseminated peritoneal adenomucinosis and from $30 \%$ to $54 \%$ for intermediate-grade disease and peritoneal mucinous carcinomatosis, with a less favorable prognosis $[10,11]$.

Our patient was treated with CRS, consisting of the removal of the enlarged appendix, wash-out of the ascites and removal of the peritoneal lesions found, in accordance with the patient's option for the most conservative procedure possible.

Related to the imaging diagnosis, a previous study including 19 patients with intraoperative diagnosis of PMP showed that in only one case the correct diagnosis of PMP was made by ultrasound, others been only partially diagnosed (encapsulated ascites, ovarian cancers, appendiceal mucocele) or misdiagnosed [4]. This case report demonstrated that PMP is a difficult imagistic diagnosis but achievable for an experienced examinator, that is trained and recognizes the ultrasonographic signs. In our case, the diagnosis was suspected after CT examination, ultrasonography described more signs of PMP and the diagnosis was eventually confirmed intraoperatively.

\section{References}

1. Smeenk RM, van Velthuysen ML, Verwaal VJ, Zoetmulder FA. Appendiceal neoplasms and pseudomyxoma peritonei: a population based study. Eur J Surg Oncol 2008; 34: 196-201.

2. Carr N, Sobin L. Tumors of the appendix. In: Bosman FT, Carneiro F, Hruban RH, Theise ND, editors. WHO Classification of tumours of the digestive system. World Health Organization classification of tumours. Vol 3. 4th ed. Lyon, France: IARC Press; 2010: 122-125.

3. Misdraji J, Yantiss RK, Graeme-Cook FM, Balis UJ, Young RH. Appendiceal mucinous neoplasms: a clinicopathologic analysis of 107 cases. Am J Surg Pathol 2003; 27: 10891103.

4. Li Y, Guo A, Tang J, Wang L, Wang J, Yu D. Role of preoperative sonography in the diagnosis and pathologic staging of pseudomyxoma peritonei. J Ultrasound Med 2013; 32: $1565-1570$

5. Que Y, Tao C, Wang X, Zhang Y, Chen B. Pseudomyxoma peritonei: some different sonographic findings. Abdom Imaging 2012; 37: 843-848.

6. Hoeffel C, Crema MD, Belkacem A, et al. Multidetector row CT: spectrum of diseases involving the ileocecal area. Radiographics 2006; 26: 1373-1390.

7. Persaud T, Swan N, Torreggiani WC. Giant mucinous cystadenoma of the appendix. Radiographics 2007; 27: 553557.

8. Tirumani SH, Fraser-Hill M, Auer R, et al. Mucinous neoplasms of the appendix: a current comprehensive clinicopathologic and imaging review. Cancer Imaging 2013; 13 : 14-25.

9. Pickhardt PJ, Levy AD, Rohrmann CA Jr, Kende AI. Primary neoplasms of the appendix: radiologic spectrum of disease with pathologic correlation. Radiographics 2003; 23: 645-662.

10. Sugarbaker PH, Chang D. Results of treatment of 385 patients with peritoneal surface spread of appendiceal malignancy. Ann Surg Oncol 1999; 6: 727-731.

11. Stewart JH $4^{\text {th }}$, Shen P, Russell GB, et al. Appendiceal neoplasms with peritoneal dissemination: outcomes after cytoreductive surgery and intraperitoneal hyperthermic chemotherapy. Ann Surg Oncol 2006; 13: 624-634.

12. Glehen O, Kwiatkowski F, Sugarbaker PH, et al. Cytoreductive surgery combined with perioperative intraperitoneal chemotherapy for the management of peritoneal carcinomatosis from colorectal cancer: a multi-institutional study. J Clin Oncol 2004; 22: 3284-3292. 